\title{
Alimentos funcionais no manejo da diabetes mellitus
}

\author{
Functional foods in the management of diabetes mellitus
}

\author{
Marília Rizzon Zaparolli ${ }^{1}$, Nayana Cavassim do Nascimento ${ }^{1}$, Deise Regina Baptista ${ }^{2}$, \\ Stela Adami Vayego ${ }^{3}$
}

\begin{abstract}
RESUMO
Objetivo: Analisar o conhecimento e o consumo de alimentos funcionais que auxiliam no controle da Diabetes mellitus em pacientes de um hospital público de Curitiba - PR.

Materiais e Métodos: Trata-se de um estudo transversal e quantitativo, realizado com pacientes diabéticos hospitalizados (Hospital de Clínicas/UFPR), no período de dezembro de 2011 a março de 2012. Para a coleta de dados foi utilizado um questionário, através do qual se obteve informações sobre o conhecimento de alimentos funcionais e consumo de cinco alimentos funcionais importantes no controle da diabetes: alho, batata yacon, cebola, farinha de casca de maracujá e linhaça. Os dados coletados foram tabulados no programa Libre Office $3.5 \AA$ e após isso se realizou análise estatística descritiva.

Resultados: Observou-se que somente 12 pacientes (35,29\%) da amostra haviam recebido informações anteriormente sobre alimentos funcionais e apenas 11 (32,35\%) responderam saber da importância desses alimentos no controle da diabetes. Dos cinco alimentos funcionais estudados, o alho e a batata yacon foram os que apresentaram maior consumo entre os diabéticos.

Conclusão: $O$ conhecimento de alimentos funcionais bem como seu consumo é pequeno por parte de pacientes diabéticos. Há a necessidade de uma maior intervenção por parte dos profissionais da Nutrição na orientação do consumo desses alimentos na dieta habitual dos diabéticos, visando promover um melhor controle da diabetes mellitus.
\end{abstract}

Palavras-chave: alimento funcional; diabetes mellitus; consumo de alimentos.

\begin{abstract}
Objective: To analyze the knowledge and the consumption of functional foods that facilitate the diabetes mellitus control in patients of a public hospital in Curitiba - PR.

Materials and Methods: This is a cross-sectional and quantitative study, conducted with diabetic patients hospitalized (Hospital de Clínicas/UFPR), in the period between December 2011 and March 2012. A questionnaire was used for data collection in order to obtain information about the knowledge of functional foods, as well as the consumption of five important functional foods for the diabetes control: garlic, yacon potato, onion, passion fruit peel flour and flaxseed. The collected data were tabulated on Libre Office $\AA 3.5$ and afterwards the analysis were performed using descriptive statistics.

Results: It was observed that only 12 patients (35.29\%) of the sample had previously received information on functional foods and only 11 (32.35\%) respondents know the importance of these foods to control diabetes. Of the five studied functional foods, garlic and potatoes yacon presented the highest consumption among diabetic patients.

Conclusion: The knowledge about functional foods, as well as its consumption by diabetic patients is small. There is a need for a greater intervention by Nutrition professionals on the orientation of these foods consumption in the usual diet of diabetic patients, aiming to promote a better control of diabetes mellitus.
\end{abstract}

Keywords: functional food; diabetes mellitus; food consumption.

\footnotetext{
${ }^{1}$ Acadêmica do Curso de Nutrição da Universidade Federal do Paraná.

${ }^{2}$ Nutricionista. Mestre em Ciências Farmacêuticas (UFPR). Docente do Departamento de Nutrição da Universidade Federal do Paraná. Coordenadora do Departamento de Nutrição da Sociedade Brasileira de Diabetes.

${ }^{3}$ Estatística. Doutora em Genética (UFPR). Docente do Departamento de Estatística da Universidade Federal do Paraná.
} 


\section{INTRODUÇÃO}

Diabetes mellitus tem sido classificada como a epidemia do século $\mathrm{XXI}$, devido ao aumento no número de diabéticos e ao seu impacto na doença cardiovascular, principal causa de morte nas sociedades desenvolvidas $^{1}$. Em 1985, estimava-se haver 30 milhões de adultos com diabetes no mundo. Entretanto, em 1995, estimou-se 135 milhões e, em 2002, esse número aumentou para 173 milhões, havendo projeção de chegar a 300 milhões em $2030^{2}$. Acredita-se que o desenvolvimento de $70 \%$ das doenças crônicas não transmissíveis está relacionado à alimentação e ao estilo de vida. A alimentação é considerada um dos aspectos mais importantes do controle e tratamento da diabetes, sendo em alguns casos, a única intervenção necessária ${ }^{3}$.

A legislação brasileira define alimentos funcionais como "todo alimento ou ingrediente que, além das funções nutricionais básicas, quando consumido como parte da dieta usual, produz efeitos metabólicos e/ou fisiológicos e/ou efeitos benéficos à saúde, devendo ser seguro para consumo sem supervisão médica"4 ${ }^{\text {. A }}$ alegação de propriedade funcional atribuída é devido à ação que substâncias presentes nos alimentos, as quais podem ou não ser nutrientes, exerce no crescimento, desenvolvimento, manutenção e outras funções do organismo humano ${ }^{5}$.

Vários estudos comprovaram que existe uma diversidade de alimentos que possuem substâncias benéficas que atuam na prevenção e/ou controle de doenças como a Diabetes e suas complicações ${ }^{6}$. Dentre eles pode-se citar: a linhaça, a batata yacon, a cebola, a farinha de casca de maracujá e o alho.

A linhaça (Linum usitatissimun) é a maior fonte de ácidos graxos ômega 3 do reino vegetal. O ômega 3 possui importante papel anti-inflamatório. Possui ainda fibras dietéticas, vitaminas $A, B, D$ e $E$ e minerais como fósforo, potássio, magnésio, cálcio e enxofre ${ }^{7}$. A ingestão de fibras aumenta a viscosidade do conteúdo gastrointestinal, diminuindo a absorção de macronutrientes, o que resulta em um aumento da sensibilidade à insulina ${ }^{8}$. Ácidos graxos essenciais, fibras e compostos fenólicos presentes na linhaça, exercem atividade antioxidante, evitando a peroxidação das membranas e consequente alteração na permeabilidade da célula e um estado de hiperglicemia com redução na tolerância à glicose. Portanto, o consumo de linhaça pode ser eficaz na redução da glicemia e melhora à tolerância da glicose ${ }^{9}$.

A batata Yacon (Smallanthus sonchifolius), diferentemente de outros tubérculos, é constituída por carboidratos que são armazenados principalmente sob a forma de frutooligossacarídeos, os quais têm algumas propriedades funcionais comprovadas, tais como redução dos níveis de colesterol e do teor de glicose sanguínea $^{10}$. Apresenta ainda em sua composição compostos fenólicos e antioxidantes ${ }^{11}$.

A farinha da casca do maracujá (Passiflora edulis flavicarpa) constitui um produto vegetal rico em fibra do tipo solúvel (pectinas e mucilagens), benéfica ao ser humano, a qual pode auxiliar na prevenção e controle de doenças ${ }^{12,13}$

A cebola (Allium cepa L.) e alho (Allium sativum L.) são alimentos amplamente utilizados para aplicações medicinais ou funcionais. São fontes de inúmeros fitoquímicos utilizados no tratamento e na prevenção de várias doenças, incluindo câncer, doença cardíaca coronária, obesidade, hipercolesterolemia, diabetes, hipertensão e distúrbios do trato gastrointestinal ${ }^{14}$. O alho possui um teor considerável de selênio agindo como antioxidante e aliina que apresenta ação hipotensora e hipoglicemiante ${ }^{15}$. Já a cebola, é rica em flavonóide quercetina, que apresenta propriedade relacionada à saúde devido ao alto poder antioxidante ${ }^{16}$.

Diante deste contexto, o objetivo deste trabalho foi analisar o conhecimento sobre alimentos funcionais e o consumo de cinco alimentos funcionais que auxiliam no controle da Diabetes mellitus em pacientes de um hospital público de Curitiba.

\section{MATERIAIS E MÉTODOS}

Trata-se de um estudo transversal e quantitativo realizado com 34 pacientes diabéticos do Hospital de Clínicas/UFPR, no período de dezembro de 2011 a março de 2012. Foram incluídos nesse estudo, pacientes diabéticos hospitalizados na Clínica Médica Feminina e Masculina do referido hospital, durante o período da coleta. Foram excluídos os pacientes 
diabéticos hospitalizados cujo estado cognitivo não permitia comunicação.

A coleta de dados iniciou após a aprovação desse estudo pelo Comitê de Ética em Pesquisa em Seres Humanos do Hospital de Clínicas/UFPR, no 2332.226/2010-09. Participaram da coleta de dados apenas os pacientes internados que compreenderam a finalidade da pesquisa, assinando ao Termo de Consentimento Livre e Esclarecido.

Para a coleta de dados, aplicou-se um questionário constituído por questões fechadas, que visaram à identificação do paciente, análise do conhecimento sobre alimentos funcionais e análise do consumo de cinco alimentos que interferem no controle da diabetes: alho, batata yacon, cebola, farinha de casca de maracujá e linhaça. O critério de escolha dos cinco alimentos pesquisados foi a prevalência destes em artigos das bases científicas Scielo, Pubmed e Lilacs quando relacionado o tema alimentos funcionais ao controle da diabetes. Estudos de revisão bibliográfica, ensaios clínicos randomizados e investigação experimental em laboratório foram utilizados. Os principais fatores protetores à diabetes citados foram os nutrientes ou fitoquímicos presentes nesses alimentos, os quais possuíam propriedade antioxidante ou antiinflamatória.

As variáveis utilizadas no estudo foram: sexo, idade, classificação do tipo de diabetes (Diabetes tipo 1, Diabetes tipo 2), conhecimento sobre alimentos funcionais, freqüência semanal de consumo dos cinco alimentos funcionais estudados, indicação do consumo e percepção de melhora no controle da glicemia após ingestão dos alimentos funcionais.

Os dados coletados foram tabulados no programa Libre Office $3.5 \AA$. Realizou-se análise estatística descritiva.

\section{RESULTADOS}

A amostra de diabéticos hospitalizados na Clínica Médica Feminina e Masculina do Hospital de Clínicas/UFPR, composta por 34 pacientes, apresentou uma média de idade de $57 \pm 14$ anos. Em relação ao sexo, 19 indivíduos (56\%) pertenciam ao sexo feminino e 15 (44\%) ao masculino. Quanto ao tipo de diabetes, a prevalência maior foi em relação ao diabetes tipo 2 , o qual foi encontrado em 30 pacientes (88\%), sendo que apenas $4(12 \%)$ eram portadores do diabetes tipo 1.

Estudando o conhecimento dos diabéticos sobre alimentos funcionais, observou-se que somente 12 sujeitos (35\%) haviam recebido informações sobre o consumo desse grupo de alimentos previamente ao estudo, enquanto que 22 (65\%) alegaram não ter recebido esse tipo de informação antes de participarem da presente pesquisa. Ao serem indagados sobre a importância de alimentos funcionais no controle glicêmico, 23 entrevistados (68\%) responderam não saber dessa propriedade funcional no controle da glicemia, enquanto que 11 (32\%) afirmaram ter conhecimento.

Ao analisar o consumo dos 5 alimentos funcionais (alho, batata yacon, cebola, farinha de casca de maracujá e linhaça) que contribuem no manejo da diabetes, o alho apresentou maior prevalência no consumo da amostra, atingindo sua totalidade (100\%). Já a farinha da casca de maracujá foi o alimento funcional com menor consumo, isto é, apenas 5 diabéticos (15\%) consumiam, enquanto que 29 (85\%) relataram não consumir esse alimento. Com resultados intermediários ao alho e a farinha de casca de maracujá, 6 sujeitos (18\%) informaram consumir batata yacon, 12 (35\%) realizavam o consumo de linhaça e 31 (91\%) consumiam cebola como parte da dieta. A frequência de consumo dos alimentos funcionais estudados está descrita na tabela 1.

Quanto à forma de consumo, o alho é consumido por todos os sujeitos na forma de tempero. Os 6 diabéticos da amostra que afirmaram consumir a batata yacon, responderam como forma de preparo a in natura; para a cebola, a resposta também foi homogênea, isto é, os 31 pacientes que fazem uso da cebola descreveram consumir na forma de salada e tempero. Dos 5 entrevistados que consomem a farinha de casca de maracujá, 3 deles $(60 \%)$ consomem a farinha misturada com as refeições e 2 (40\%) consomem na forma pura. Em relação à linhaça, 10 pessoas (83\%) relataram consumir na forma de farinha e $2(17 \%)$ na forma de grão, ambos misturados nas refeições.

Em relação à indicação do consumo de alimentos 
funcionais para o controle da diabetes, 22 indivíduos (65\%) responderam que não houve indicação, 5 (15\%) alegaram que a indicação foi realizada por profissionais da saúde, 3 (9\%) que a indicação foi realizada pela mídia, 3 (9\%) que a indicação foi realizada por conhecidos e 1 indivíduo (2\%) respondeu que a indicação foi realizada por familiares.

Ao final da entrevista, os pacientes foram questionados se houve melhora na glicemia após o consumo dos alimentos funcionais. Do total da amostra, 16 diabéticos (47\%) não souberam informar se houve melhora ou não, 5 (15\%) relataram que não houve melhora e 13 (38\%) relataram que houve melhora na glicemia.

\section{DISCUSSÃo}

Os 34 pacientes diabéticos entrevistados haviam consumido pelo menos um dos alimentos com alegação de propriedade funcional estudados para controle da diabetes. Entretanto, ao observar-se o consumo isolado dos alimentos estudados, verificou-se que o consumo ainda é baixo para esse grupo de alimentos nessa amostra de indivíduos. Além disso, nesse estudo, observou-se que alguns indivíduos fazem o uso de alimentos funcionais sem saber da sua propriedade.

Um estudo realizado por Quadros e Baptista ${ }^{17}$ mostrou que de uma amostra de 83 pacientes diabéticos, 60 desses (72,29\%) haviam consumido algum tipo de alimento ou produto com possíveis efeitos no controle da glicemia. Brito e Baptista ${ }^{18}$ demonstraram que, dos 40 entrevistados, apenas 14 pacientes (35\%) responderam que haviam consumido algum alimento para controle glicêmico. Com base nesses estudos, observa-se que o consumo de alimentos funcionais por diabéticos ainda é baixo e pode estar associado com a falta de conhecimento sobre alimentos funcionais, o que foi verificado no presente estudo, e a falta de divulgação sobre a importância de alguns desses alimentos no controle da doença.

Ainda em relação aos resultados apresentados por Brito $^{18}$, dos 14 pacientes que afirmaram já ter usado algum alimento com possível efeito no controle da glicemia, 25,5\% citaram o chá de pata-de-vaca; seguido por farinha de casca de maracujá (15,7\%); batata yacon
(13,7\%); chá de quebra-pedra (11,8\%); chá de berinjela $(11,8 \%)$. O restante se distribui entre chá de cebola e/ou alho, chá de limão, entre outros (chá de jambolão). Nos resultados de Quadros e Baptista ${ }^{17}$, a pata-de-vaca foi o alimento que apresentou maior frequência de consumo no grupo (50\%), seguido da batata yacon $(41,67 \%)$. Já o alho e a cebola apresentaram uma frequência relativamente baixa $(3,33 \%)$. Nesse aspecto, esses estudos diferem do presente, no qual a maior prevalência de relato de consumo foi em relação ao alho e a cebola. Além disso, a linhaça consumida por 12 pacientes $(35,29 \%)$ não foi citada pelos pacientes nos dois estudos. A farinha de casca de maracujá foi citada como segundo alimento mais consumido no estudo realizado por Brito e Baptista $(2005)^{18}$ divergindo do presente estudo no qual foi a menos citada. Entretanto, as divergências encontradas entre esses estudos podem estar relacionadas aos hábitos alimentares diversificados entre as amostras de pacientes estudadas.

Brito e Baptista ${ }^{18}$ observaram que nenhum alimento funcional havia sido indicado por algum profissional da saúde, segundo os diabéticos entrevistados. Resultado que se assemelha com o encontrado nesse estudo, no qual apenas 5 indivíduos (14,71\%) responderam que a indicação foi realizada por profissionais da saúde. Com isso, observa-se que a atuação do profissional da saúde, especialmente da Nutrição, ainda é escasso no que se diz respeito à divulgação do conhecimento sobre alimentos funcionais, principalmente daqueles que possuem alguma propriedade de controle glicêmico em diabéticos.

Um estudo realizado por Millone et al. ${ }^{19}$, no qual foi investigado o conhecimento de graduandos em Nutrição sobre alimentos funcionais e suas propriedades, concluiu que somente $50 \%(n=57)$ dos profissionais tinham um conhecimento prévio sobre o assunto, sendo que $63 \%$ destes profissionais não sabiam a definição correta de alimentos funcionais. Quadros e Baptista ${ }^{17}$ ainda demonstram que, dos 60 pacientes diabéticos que alegaram consumir algum produto com 0 intuito de melhorar a glicemia, apenas 28 (46,67\%) relataram mudanças no seu estado de saúde quanto a diabetes, o que se assemelha com o resultado encontrado nesse 
TABELA 1 - Frequência de consumo de alimentos funcionais importantes para o controle glicêmico pelos diabéticos.

\begin{tabular}{|c|c|c|}
\hline Frequência & Número & Percentual \\
\hline \multicolumn{3}{|l|}{ Alho } \\
\hline Todos os dias & 31 & 91 \\
\hline $2-3$ vezes por semana & 2 & 6 \\
\hline $4-5$ vezes por semana & 1 & 3 \\
\hline \multicolumn{3}{|l|}{ Batata yacon } \\
\hline Todos os dias & 2 & 33 \\
\hline 1 vez por semana & 3 & 50 \\
\hline $2-3$ vezes por semana & 1 & 17 \\
\hline \multicolumn{3}{|l|}{ Cebola } \\
\hline Todos os dias & 27 & 88 \\
\hline $4-5$ vezes por semana & 2 & 6 \\
\hline $2-3$ vezes por semana & 2 & 6 \\
\hline \multicolumn{3}{|c|}{ Farinha da casca de maracujá } \\
\hline Todos os dias & 2 & 40 \\
\hline 2-3 vezes por semana & 1 & 20 \\
\hline 1 vez por mês & 1 & 20 \\
\hline $2-3$ vezes por mês & 1 & 20 \\
\hline \multicolumn{3}{|l|}{ Linhaça } \\
\hline Todos os dias & 8 & 67 \\
\hline 1 vez por semana & 1 & 8 \\
\hline $2-3$ vezes por semana & 1 & 8 \\
\hline 1 vez por mês & 2 & 17 \\
\hline
\end{tabular}

nesse estudo, no qual apenas 13 diabéticos (38,24\%) relataram que houve melhora na glicemia. Essa taxa baixa de melhora na glicemia não significa que os alimentos não possuem efeito sobre o controle glicêmico, mas pode estar relacionada a uma quantidade de consumo insuficiente do alimento, não exercendo sua propriedade funcional.

Alguns alimentos funcionais possuem uma recomendação diária comprovada para exercerem a propriedade funcional. A American Dietetic Association ${ }^{20}$ estabeleceu que o consumo de 1 dente de alho por dia, o que corresponde a 600-900 mg, já é o suficiente para que esse alimento exerça sua propriedade funcional. Em relação à batata Yacon foi constatado que a ingestão de 15,44 g/dia de farinha ou $267,8 \mathrm{~g} / \mathrm{dia}$ da polpa da raiz seriam suficientes para fornecer a quantidade de FOS necessária para alegação funcional na Diabetes mellitus ${ }^{21}$. Quanto à cebola, o consumo de
$60 \mathrm{~g} /$ dia reduziu significativamente os níveis de glicose em pacientes diabéticos ${ }^{22}$. A suplementação com $30 \mathrm{~g} /$ dia de farinha de casca de maracujá durante 60 dias exerceu uma melhora significativa nos índices glicêmicos desses indivíduos e a suplementação de $10 \mathrm{~g} /$ dia de semente de linhaça durante um mês em pacientes com diabetes tipo 2, apresentou redução de glicose de jejum e hemoglobina glicada, além de uma redução no colesterol total e triglicérides ${ }^{24}$.

É importante ressaltar que existem inúmeras variáveis que interferem na glicemia e que a quantidade consumida desses alimentos deve ser analisada com cautela, junto a um plano alimentar individualizado às necessidades de cada paciente.

No presente estudo, não se pode considerar o efeito na glicemia como sendo algo concreto, uma vez que o estudo não avaliou a quantidade consumida dos alimentos estudados. Ressalta-se ainda que o efeito funcional, muitas vezes, acaba não sendo efetivo, devido à descontinuidade do uso desses alimentos. A falta de persistência no tratamento dietoterápico costuma ser um impedimento difícil de ser combatido. Alguns diabéticos afirmam saber os cuidados que teriam que ter com relação à alimentação para controlar a doença, porém não os têm regularmente ${ }^{25}$.

As evidências científicas têm mostrado que alguns alimentos possuem benefícios potenciais para a saúde, atuando principalmente na prevenção e controle de doenças crônicas não transmissíveis, resultantes do hábito alimentar das sociedades modernas. Nesse estudo, observou-se que a amostra de diabéticos apresenta baixo consumo e conhecimento de alimentos funcionais importantes no controle glicêmico. Isso pode ser explicado pelo fato de alimentos funcionais ser um tema novo e pouco explorado no nosso país. Além disso, deve-se considerar que muitas das evidências atuais sobre alimentos funcionais são desprovidas de ensaios clínicos bem estabelecidos, o que dificulta a capacitação dos profissionais da saúde e a divulgação entre a população. Há a necessidade de uma maior intervenção por parte dos profissionais da Nutrição na orientação do consumo desses alimentos na dieta habitual dos diabéticos, visando promover um melhor controle da diabetes mellitus. 


\section{REFERÊNCIAS}

1. Valdés S, Rojo-Martínez G, Soriguer F. Evolución de la prevalencia de la diabetes tipo 2 en población adulta española. Med Clin. 2007;129(9):352-5.

2. Wild S, Roglic G, Green A, Sicree R, King H. Global prevalence of diabetes - estimates for the year 2000 e projections for 2030. Diabetes Care. 2004;27(5):1047-53.

3. Castell GS, Sagnier LB. Larousse da dieta e da Nutrição. São Paulo :Larousse do Brasil; 2004.

4. Brasil. Agência Nacional de Vigilância Sanitária. Portaria no 398. Brasília: Ministério da Saúde; 1999.

5. American Dietetic Association. Position of the American Dietetic Association: functional foods. Am J Diet Assoc. 2004;104:814-26.

6. Basho SM, Bin MC. Propriedades dos alimentos funcionais e seu papel na prevenção e controle da hipertensão e diabetes. Interbio. 2010;4(1):48-58.

7. Oliveira TM, Pirozi MR, Borges JTS. Elaboração de pão de sal utilizando farinha mista de trigo e linhaça. Alim Nutr. 2007 Abr/June;18(2):141-50.

8. Burton-Freeman B. Dietary fiber and energy regulation. $J$ Nutr. 2000;130:272-5.

9. Galvão EL, Silva DCF, Silva JO, Moreira AVB, Sousa EMBD. Avaliação do potencial antioxidante e extração subcrítica do óleo de linhaça. Ciênc Tecnol Aliment. 2008 jul/set;28(3):551-7.

10. Santana I, Cardoso MH. Raiz tuberosa de yacon (Smallanthus sonchifolius): potencialidade de cultivo, aspectos tecnológicos e nutricionais. Ciênc Rural 2008; 38(3):898-905

11. Sales RL, Rodrigues FC, Costa NMB, Ferreira CLLF. Alimentos funcionais: componentes bioativos e efeitos fisiológicos. Rio de Janeiro: Rubio; 2010.

12. Cordova KRV, Gama TMMTB, Winter CMG, Kaskantizis G, Freitas RJS. Características físico-químicas da casca do maracujá-amarelo (Passiflora edulis Flavicarpa degener) obtida por secagem. B CEPPA. 2005;23(2):22130.

13. Camargo P, Moraes C, Schemberger A, Santos CP, Schemin MHC. Rendimento da Pectina na Casca do Maracujá em seus estágios diferentes de maturação: verde, maduro e senescência. Série em Ciência e Tecnologia de Alimentos: agroindústria, energia e meio ambiente [periódico online]. 2008 [capturado 2011 May 25]; Disponível em: http://www.pg.cefetpr.br/coali/livro/ volume2/artigos/009.pdf

14. Lanzotti V. The analysis of onion and garlic. J Chromatogr A. 2006;1112:3-22.

15. Corzo-Martínez M; Corzo N, Villamiel M. Biological properties of onions and garlic. Trends Food Sci Tech. 2007 Dec;18(12):609-25.

16. Leite DL, Santos ACA, Bertussi R. Concentração do flavonóide quercetina em quatro genótipos de cebola. Hortic Bras 2008;26(2):6661-4.

17. Quadros DA de; Baptista, DR. Perfil dos diabéticos associados da APAD - Consumo de alimentos funcionais e/ou alternativos como uma terapia de controle do diabetes mellitus (monografia). Curitiba; 2002.

18. Brito TM de; Baptista, DR. Perfil dos pacientes diabéticos internados no Hospital de Clínicas da Universidade Federal do Paraná - UFPR e avaliação do consumo de alimentos funcionais como controle do diabetes mellitus (trabalho de conclusão de curso). Curitiba; 2005.

19. Millone MV, Olagnero GF, Santana EC. Alimentos funcionales: análisis de la recomendación em La práctica diária. Diaeta. 2011 Mar:29(134):7-15.

20. American Dietetic Association. Position of the American Dietetic Association: Functional foods. Am J Diet Assoc.199;10(1):1278-84.

21. Vasconcelos CM, Silva CO da, Teixeira LJQ, Chaves JBP, Martino HSD. Determinação da fração da fibra alimentar solúvel em raiz e farinha de yacon (Smallanthus sonchifolius) pelo método enzimático-gravimétrico e cromatografia líquida de alta eficiência. Rev Inst Adolfo Lutz. 2010;69(2):188-93.

22. Tjokroprawiro A, Pikir BS, Budhiarta AA, Pranawa, Soewondo $H$, Donosepoetro $M$, Budhianto FX, Wibowo JA, Tanuwidjaja SJ, Pangemanan M. Metabolic effects of onion and green beans on diabetic patients. Tohoku J Exp Med.1983;141:671-6.

23. Janebro DI, Queiroz MSR de, Ramos AT, Sabaa-Srur AUO, Cunha MAL da, Diniz MFFM. Efeito da farinha da casca do maracujá-amarelo (Passiflora edulis f. flavicarpa Deg.) nos níveis glicêmicos e lipídicos de pacientes diabéticos tipo 2. Rev Bras Farmacogn 2008;18(0):724-32.

24. Ceolin J, De Biase LS. Conhecimento dos diabéticos a respeito da doença e da realização do autocuidado. Perspectiva. 2011;35(129):143-56.

25. Mani UV, Mani I, Biswas M, Kumar SN. An open-label study on the effect of flax seed powder (Linum usitatissimum) supplementation in the management of diabetes mellitus. J Diet Suppl. 2011 Sep;8(3):257-65

\section{Endereço para correspondência:}

Marília Rizzon Zaparolli

Rua Doutor Pedrosa ㄲo194

Curitiba/Paraná - CEP 80420-120

Telefone: +55 4130952633

E-mail: mariliazaparolli@gmail.com 